\title{
EVALUATION OF CLINICAL OUTCOME OF MEDIAL PATELLOFEMORAL LIGAMENT RECONSTRUCTION IN PATIENTS WITH RECURRENT PATELLAR INSTABILITY
}

\author{
Sher Bahadur Singh ${ }^{1}$, Hiranmoy Deb², Emdad Hossain³, Kaushik Banerjee ${ }^{4}$ Ranjit Shaw 5 \\ 1 Senior Resident, Department of Orthopaedics, South Howrah State General Hospital, Howrah, West Bengal, India. \\ ${ }^{2}$ Assistant Professor, Department of Orthopaedics, R.G. Kar Medical College, Kolkata, West Bengal, India. \\ ${ }^{3}$ Associate Professor, Department of Orthopaedics, Purulia Government Medical College, Purulia, West-Bengal, India \\ ${ }^{4}$ Professor, Department of Orthopaedics, R. G, Kar Medical College, Kolkata, India. \\ ${ }^{5}$ Assistant Professor, Department of Orthopaedics, $R$ G Kar Medical College, Kolkata, India.
}

\begin{abstract}
BACKGROUND
Recurrent patellar instability is not an uncommon entity with acute patellar dislocation accounting for $2 \%$ to $3 \%$ of all knee injuries and is the second most common cause of traumatic knee haemarthrosis. Based on recent biomechanical studies, among the medial patellar stabilisers the Medial Patellofemoral Ligament (MPFL) is considered as the primary restraint against lateral dislocation of the patella.
\end{abstract}

ABSTRACT

\section{MATERIALS AND METHODS}

This is an observational study. 25 patients (15 males and 10 females) with recurrent patellar dislocation were operated with MPFL reconstruction with tendon graft and bio screw. Their clinical outcome was measured with respect to pre-op, 3 months post-op and 6 months post-op Kujala score. SPSS software was used for analysis.

\section{RESULTS}

Pre-op mean Kujala score was 71.24 and 6 months mean Kujala score was 75.12.

\section{CONCLUSION}

This study demonstrates that clinical outcomes are good after isolated MPFL reconstruction, as evidenced by the Kujala score. Study findings correlate well with other previous studies done on MPFL reconstruction for recurrent patellar dislocation.

\section{KEY WORDS}

MPFL, Recurrent Patellar Dislocation, Kujala Score.

HOW TO CITE THIS ARTICLE: Singh SB, Deb H, Hossain E, et al. Evaluation of clinical outcome of medial patellofemoral ligament reconstruction in patients with recurrent patellar instability. J. Evolution Med. Dent. Sci. 2018;7(36):3968-3973, DOI: $10.14260 /$ jemds/2018/887

\section{BACKGROUND}

Recurrent patellar instability is not an uncommon entity with acute patellar dislocation accounting for $2 \%$ to $3 \%$ of all knee injuries ${ }^{1}$ and is the second most common cause of traumatic knee hemarthrosis. ${ }^{2}$ Patellar injury and dislocation are more prevalent in individuals who participate in sports activities. Anterior knee pain is the most common initial manifestation. It can follow a violent blow to normal knee or trivial trauma in an abnormally predisposed knee. Patellofemoral dysfunction is more common in females than in males. Patellofemoral instability is mostly found in females after an initial episode of acute patellar dislocation. Among the people who has an initial dislocation of patella, the risk of subsequent dislocation will be higher. ${ }^{3}$ Various factors are attributed with recurrent dislocations of patella. Static stabilisers of patella are shape of patella, femoral sulcus, length of patellar tendon and normally tensioned medial capsule reinforced by patellofemoral and patellotibial ligament. The other factors of acute patellar and recurrent

'Financial or Other Competing Interest': None.

Submission 28-06-2018, Peer Review 19-08-2018,

Acceptance 25-08-2018, Published 03-09-2018.

Corresponding Author:

Dr. Hiranmoy Deb,

Greenwood Park, Flat No. HIG 1/2 4B,

New Town, Kolkata-700156, West Bengal, India.

E-mail: firstnamehiranmaydeb@yahoo.com

DOI: $10.14260 /$ jemds $/ 2018 / 887$ patellar dislocations are high-riding patella i.e. patella alta, increased Q-angle with lateral position of the tibial tuberosity, genu valgum, ligamentous hyperlaxity, vastus medialis muscle hypoplasia, external tibial torsion, subtalar joint pronation and increased femoral anteversion. 4,5 Those patients who have suffered acute patellar dislocation are prone to subsequent dislocations. ${ }^{3}$ Long term complications of acute patellar dislocations are pain, patellar instability, recurrent dislocations, decreased level of sporting activity and patellofemoral arthritis.6,7 The treatment of ligamentous injuries and chondral lesions is best judged by MR imaging. ${ }^{8}$ The Medial Patellofemoral Ligament (MPFL) is the primary restraint against lateral dislocation of the patella. ${ }^{9,10,11}$ Conlan et $\mathrm{al}^{9}$ reported that the MPFL provided $53 \%$ of the total medial restraining force. Hautamaa et $\mathrm{al}^{11}$ and Desio et al ${ }^{10}$ respectively reported $55 \%$ and $60 \%$. The MPFL extends from the superior two-thirds of the medial patellar margin to a point distal to the adductor tubercle and proximal to the posterior aspect of the femoral attachment of the medial collateral ligament. The superficial fibres blend with the posterior capsule of the knee. Though several surgical techniques for treatment of patellar instability has been described, much debate still remains about the best outcome.12,13,14,15 Studies of different MPFL reconstruction techniques have been done to compare the clinical outcome of symptoms and patellofemoral congruency. 16,17,18,19,20,21,22,23 


\section{Aim of the Study}

The aim of the study is to evaluate the overall outcome, both subjective and objective, of Medial Patellofemoral Ligament (MPFL) reconstruction in cases of recurrent patellar dislocations.

\section{MATERIALS AND METHODS}

This study was approved by Institutional Ethical Committee

\section{Study Design}

This is an Observational Study.

\section{Study Area}

Department of Orthopaedics, RG Kar Medical College and 25 (15 males and 10 females) mean age patients were included in the study, who had history of recurrent patellar dislocation as per inclusion and exclusion criteria. All patients were analysed at regular intervals and raw data were obtained. Shapiro-Wilk test of normality applied as the sample size was small. SPSS software was used for analysis.

\section{Study Period}

January 2016 to December 2017.

\section{Study Population}

Indoor and outdoor patients presenting with history of or clinical features of patellar dislocation.

\section{Inclusion Criteria}

Recurrent patellar dislocations in skeletally mature patients.

\section{Exclusion Criteria}

1. Patients with excessive femoral anteversion.

2. Patients with external tibial torsion.

3. Patients with genu valgum.

4. Previously operated knee.

5. Patients with any bony abnormality in patella, femur or tibia.

6. Patients with pre-existing knee symptoms except knee dislocation and pain.

Subjects were sub-divided on the basis of sex, age, sports activities and associated deformity (presence of patella alta and femoral anteversion $>20^{\circ}$ ). Patients were evaluated using Kujala Scoring Questionnaire pre-operatively at 3 months post-operatively and 6 months post-operatively.

\section{Pre-Operative Planning}

Patients attending our hospital with history of knee injury were examined and those diagnosed with two or more episodes of patellar dislocation following trauma were included in the study.

Numerous clinical tests were done followed by radiological investigations to establish and rule out other pathologies.

\section{Clinical}

Patellar apprehension test, active and dynamic patellar tracking, Q-Angle, femoral anteversion measurement, patellar grind test, patellar gliding, patellar tilt test, power of the quadriceps, measurement of thigh foot angle, generalised ligament laxity using Beighton score was done.

\section{Radiological}

Initial radiographs in true AP, lateral, axial and skyline views were taken to see patellar position, Blumensaat line, InsallSalvati index, trochlear depth (Dejour), patellar height (Caton Deschamps), Blackburne-Peel ratio, crossing sign and trochlear bump. CT scan was done to measure TT-TG distance and MRI was done to know the status of soft tissue structures including MPFL, quadriceps and intra-articular structures. Patients were selected as per inclusion and exclusion criteria as previously fixed. Patients were sent for anaesthetic fitness and then MPFL reconstruction was planned and posted for surgery.

\section{Operative Procedure}

Patient was placed in supine posture with limb hanging from the edge of the operation table from mid-thigh region. Anaesthesia, spinal or general was given and pneumatic tourniquets were applied followed by antiseptic scrubbing and draping.

Arthroscopic evaluation of the knee joint was done using standard anterolateral and anteromedial portals. Undersurface of the patella was visualised with flexion and extension of the knee to assess patellar tracking. Other ligaments and menisci were also seen.

After this, graft was harvested from patient's own hamstrings. Semitendinosus was harvested from the same limb. After the graft was sized, it was secured in normal saline and gentamycin. Two small incisions were made, one just beneath and off the superior pole of patella and another starting at the adductor tubercle and ending just distal to the medial epicondyle of the femur.

Subcutaneous dissection was done to expose proximal medial retinaculum at its insertion into the proximal medial portion of the patella, after which it is incised about $1.5 \mathrm{~cm}$ using blunt dissection space was made between layer 2 and 3 (between MPFL and capsular layer) up to the medial femoral epicondyle. The second incision was made in the saddle area between the adductor tubercle and medial epicondyle.

A superficial trough along the medial aspect of patella along the proximal half was done centering between the articular surface and cortex. After this, two suture anchors were placed in the trough, one just proximal to the midpatella and another just distal to the tip of superior pole of patella keeping the knee in 45-degree flexion directed in the strong cancellous bone.

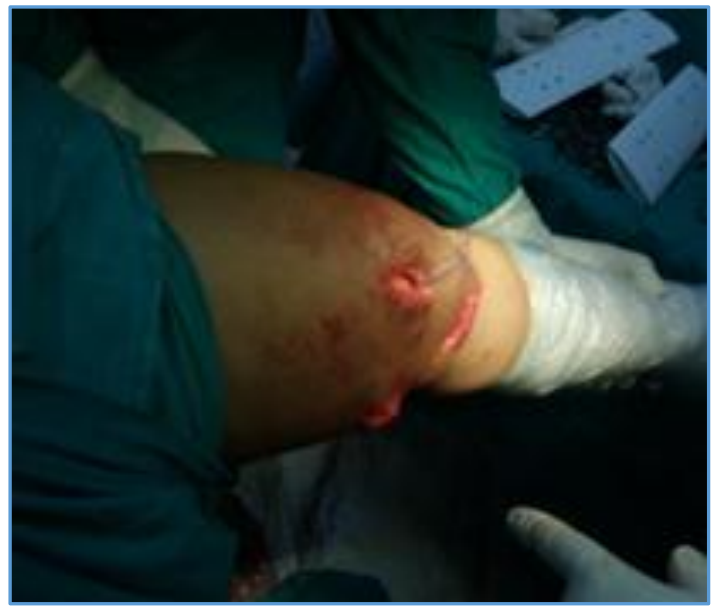

Figure 1. Suture Anchors seen fixed to Patella 
Now site for femoral tunnel was marked approximately 1 $\mathrm{cm}$ distal and $5 \mathrm{~mm}$ posterior to the adductor tubercle, slight proximal to the medial epicondyle. Confirmation was done using C-arm. A beathpin guidewire was placed at this spot and then tail of the suture anchor was passed through the soft tissue trough made to the area of the guidewire. Knee was moved through 0 to 70 degrees of flexion and tension in the tails of the anchor was observed.

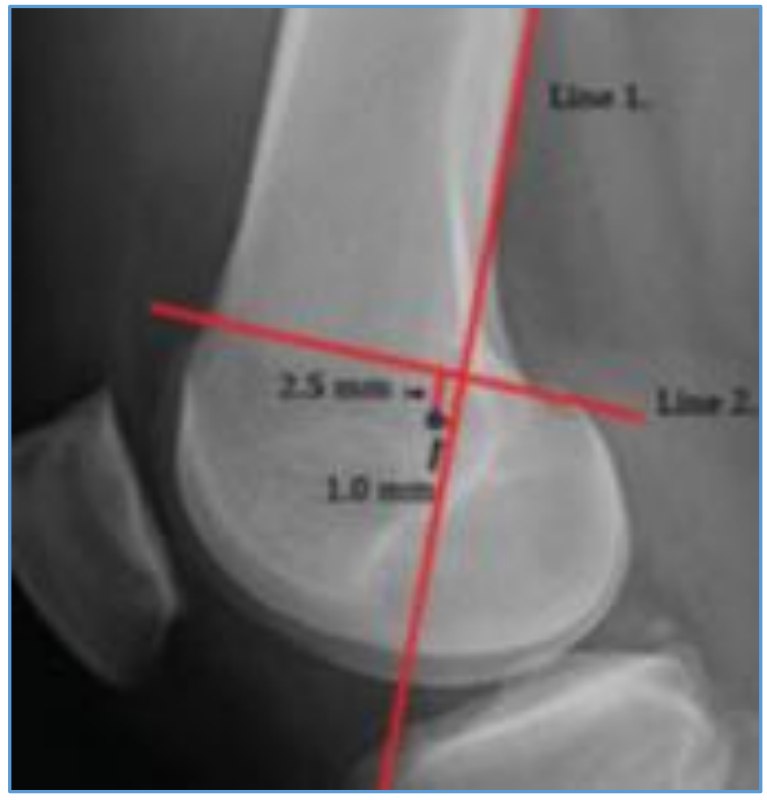

Figure 2. Exact Location of Femoral attachment Site

The length of graft was measured, ensuring it was greater than $16 \mathrm{~cm}$ and loop of the graft was secured with the two suture anchors in the patellar trough. The tails of the graft were then passed through layer 2 and 3 . At the selected site of femoral tunnel, beathpin was advanced laterally to pass the skin. A femoral tunnel was made with diameter depending upon the thickness of the graft using beathpin as guidewire.

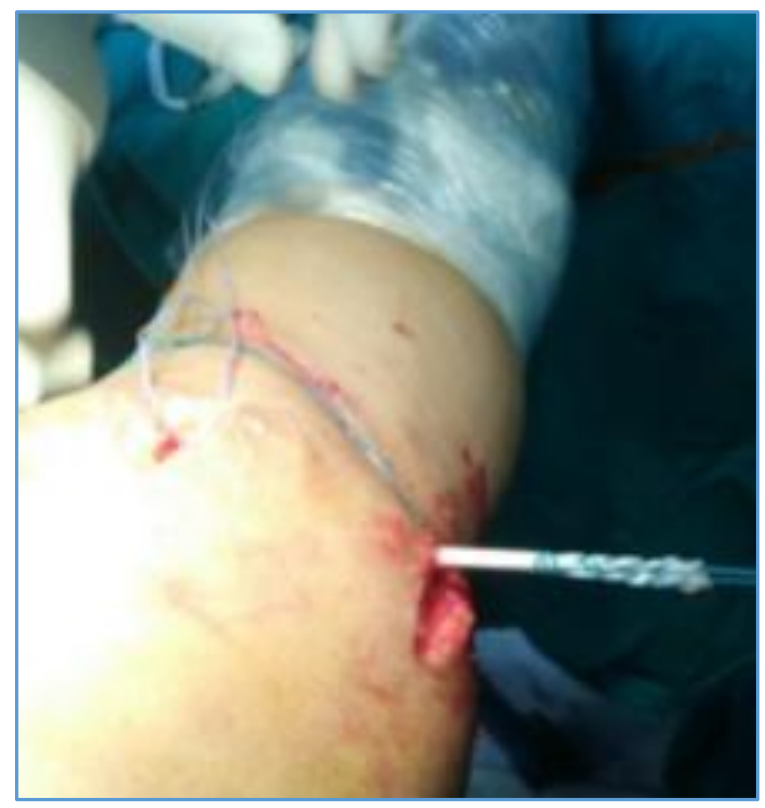

Figure 3. Graft pulled out from Femoral Incision Site after passing Layer 2
The whip stitch attached at the two tails of the graft were then placed in the eye of the beathpin and pulled out laterally. After this and before fixing the graft using the composite bioscrew, knee was again flexed and extended to ensure appropriate tensioning. Passive gliding at 30 degrees of flexion should ideally be upto two quadrants. After which the graft was fixed using Biocomposite screw, $1 \mathrm{~mm}$ smaller than the tunnel size.

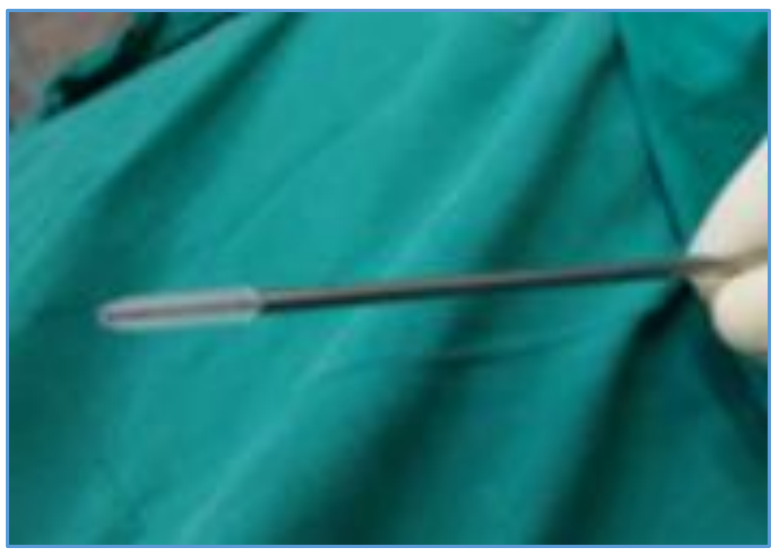

Figure 4. Biocomposite Screw

After fixation, knee was again moved to ensure the final position. Retinaculum was repaired followed by subcutaneous layer and skin. Sterile compression dressing was applied, tourniquet was deflated and limb was placed in a long knee extension brace.

\section{Post-Operative Care Immediate}

1. Knee was immobilised in the brace initially.

2. Dressing was changed 48 hours post-operatively.

3. Stitches were removed on $14^{\text {th }}$ post-operative day.

4. Ice packs were applied to decrease post-surgical swelling and effusion.

\section{Rehabilitation at 0 - 6 Weeks}

1. Weight bearing- Partial weight bearing with crutches and knee extension brace was allowed after 2 weeks, full weight bearing with brace after 4 weeks.

2. Brace application protocol-

- 0 - 2 weeks- Locked in extension.

- 2 - 4 weeks- Unlocked for exercises only.

- 4 - 6 weeks- Unlocked during sleeping.

3. Exercise protocol-

- Quadriceps sets both supine and prone.

- Four-way leg lifts with brace-on, in supine for hip strength.

- Ankle pumps.

- Ankle isotonic with exercise band.

- Core training in appropriate positions.

- Gastrocnemius/soleus stretches.

- Hamstrings stretches.

\section{After 4 - 6 Weeks}

- Partial wall squats 0 - 45․

- Double leg heel raise.

- ROM achieved from $0^{\circ}-90^{\circ}$ at the end of 6 weeks. 


\section{Rehabilitation at 6 - 8 Weeks}

- Full weight bearing with brace unlocked.

- Progress to full ROM.

- Continue all exercises.

- Double limb balance training, gradually shifted to single limb.

\section{Rehabilitation at 8 Weeks - 4 Months}

- Full weight bearing with brace discontinued.

- Continue previous exercises.

- Step-up's start with increase in height.

- Partial lunges in sagittal plane.

- Partial squats with resisted terminal knee extension.

\section{Statistical Analysis}

Statistical analysis was done using SPSS Version 16.0. Paired ' $\mathrm{t}$ ' test was applied to compare means of Kujala score at different phases.

\section{RESULTS}

We wanted to compare the Kujala score values at pre-op, 3 months post-op and 6 months post-op, so Shapiro-Wilk test of normality was applied as the sample size was small.

For assesment of distribution pattern of Kujala score at three different phases, Shapiro-Wilk test was applied. Test results showed observation at three different phases to be of normal distribution pattern ( $p>0.05)$. Paired ' $t$ ' test was applied to compare mean scores of three different phases.

\begin{tabular}{|c|c|c|c|}
\hline Variables & Mean & $\begin{array}{c}\text { Standard } \\
\text { Deviation }\end{array}$ & $\begin{array}{c}\text { Shapiro-Wilk Test } \\
\text { for Normality } \\
\text { (P-value) }\end{array}$ \\
\hline $\begin{array}{c}\text { Pre-op Kujala } \\
\text { score }\end{array}$ & 71.24 & 7.195 & .349 \\
\hline $\begin{array}{c}\text { Kujala score at 3 } \\
\text { months }\end{array}$ & 67.28 & 8.269 & .187 \\
\hline $\begin{array}{c}\text { Kujala score at 6 } \\
\text { months }\end{array}$ & 75.12 & 9.466 & .124 \\
\hline $\begin{array}{c}\text { Table 1. Distribution pattern of observation on Kujala } \\
\text { Score }\end{array}$ \\
\hline \multicolumn{3}{|c}{} \\
\hline
\end{tabular}

\begin{tabular}{|c|c|c|c|}
\hline Kujala Score & T value & $\begin{array}{c}\text { Df (Degree of } \\
\text { Freedom) }\end{array}$ & P value \\
\hline $\begin{array}{c}\text { Pre-operative and 3 } \\
\text { months interval }\end{array}$ & 6.775 & 24 & .000 \\
\hline $\begin{array}{c}\text { Pre-operative and 6 } \\
\text { months interval }\end{array}$ & -5.341 & 24 & .000 \\
\hline $\begin{array}{c}\text { Post-operative 3 months } \\
\text { and 6 months interval }\end{array}$ & -19.666 & 24 & .000 \\
\hline \multicolumn{4}{|c|}{ Table 2. Average Kujala Score at different Phases } \\
\hline
\end{tabular}

There was marked improvement in clinical outcome after MPFL reconstruction at 6 months.

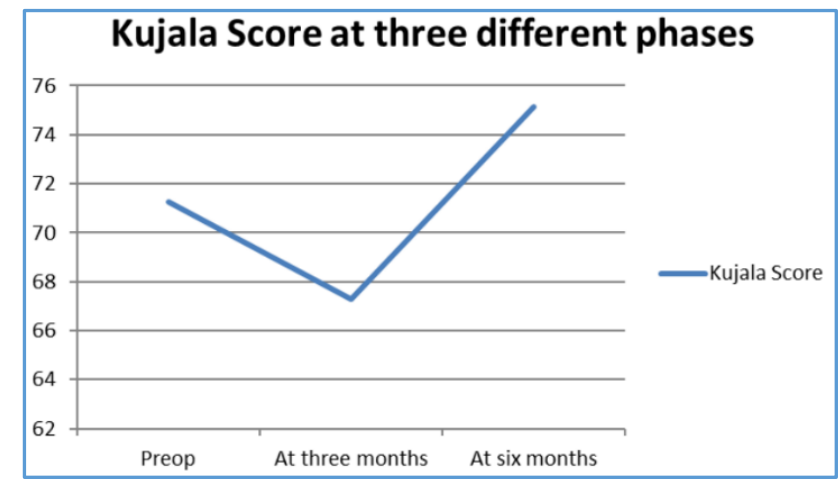

Figure 5. Profile Plots of Mean Kujala Score at 3 Intervals

The difference between the pre-op and 6 months interval, Kujala score was compared in two age groups i.e. 18 - 30 years and $>30$ years' age group, which showed that the median difference in case of 18 - 30 years' age group was more as compared to $>30$ years' age group. But, this difference was not found to be statistically significant.

The difference between the pre-op and at 6 months interval Kujala score was compared in two sex groups, which showed that the median difference in case of male group was more as compared to female group. But, this difference was not found to be statistically significant.

The difference between the pre-op and at 6 months interval Kujala score was compared in between sports persons and non-sports persons group, which showed that the median difference in case of sports persons group was more as compared to non-sports persons group. But, this difference was not found to be statistically significant.

The difference between the pre-op and at 6 months Kujala score was compared in two groups i.e. presence or absence of deformity, which showed that the median difference in case of deformity present group was more as compared to deformity absent group. And also, this difference was found to be statistically significant.

\section{DISCUSSION}

The study was conducted in the Department of Orthopaedics, RG Kar Medical College and Hospital, Kolkata. The study period was from January 2016 to September 2017. A total of 25 patients with diagnosis of recurrent patellar dislocation were included in the study. Clinical and radiological work-up was done for selection of cases as per inclusion and exclusion criteria. After the selection of appropriate candidates, interventional longitudinal prospective study was done. There was no loss of any patient in follow-up and the final results were analysed at the end of the study.

Total number of males in the study were 15 , whereas females were 10 in number. 14 were in the age group of 18 30 years, 8 were in the age group of $30-45$ years and 3 were above 45 years. 6 people were actively involved in sports activities and 6 people had some deformity present. Pre-op assessment was done and pre-op Kujala score was recorded for every patient.

They were posted for MPFL reconstruction using autologous semitendinosus graft from the same limb and fixed with suture anchors and bioabsorbable screw. No postoperative complications in the form of infection were encountered. A well-guided rehabilitation program was 
started, and patients were assessed again at 3 and 6 months post-operatively and Kujala knee scores were noted.

The Kujala scores were then analysed to obtain the results at completion of our study. The means of pre-op Kujala score, 3 months post-op Kujala score and 6 months post-op Kujala scores were $71.24,67.28$ and 75.12 respectively.

There was statistically significant difference noted on comparison of the scores at all the three intervals. Kujala score at 3 months post-op was found to be less than pre-op Kujala score and it was statistically significant. Kujala score at 6 months post-op was found to be more than preop score and it was found to be statistically significant.

The difference between the pre-op and at 6 months Kujala score was compared in two age groups, i.e. 18 - 30 years and $>30$ years, which showed that the Median difference in case of 18 - 30 years' age group was more as compared to $>30$ years' age group.

The difference between the pre-op and at 6 months Kujala score was compared in two sex groups, which showed that the median difference in case of males was more as compared to females.

The difference between the pre-op and at 6 months Kujala score was compared in between sports persons and non-sports persons group, which showed that the Median difference in case of sports persons group was more as compared to non-sports persons group.

But the above three comparisons (age, sex, sports group) were not statistically significant when analysed using nonparametric tests.

The difference between the pre-op and at 6 months Kujala score was compared in two groups i.e. presence or absence of deformity, which showed that the Median difference in case of deformity present group was more as compared to deformity absent group and it was also statistically significant.

\section{CONCLUSION}

This study demonstrates that clinical outcomes are good after isolated MPFL reconstruction as evidenced by the Kujala score. Study findings correlate well with other previous studies done on MPFL reconstruction for recurrent patellar dislocation. A wide range of predisposing factors for patellar dislocation has been overlooked while selecting patients for this procedure, for example specific anatomic features such as a normal Q-angle, lack of severe trochlear dysplasia, TT-TG distance and patellar height.

In short, more or less normal knees with history of trauma were included in the study. Given the efficacy of isolated MPFL reconstruction, future investigations should aim to establish more uniform criteria for selecting patients to undergo this procedure. Studies should also include clinical tests alongside knee outcome scores and should also incorporate long-term clinical outcomes to further strengthen the results.

\section{REFERENCES}

[1] Aglietti P, Buzzi R, Insall J. Disorders of the patellofemoral joint. In: Insall J, Scott W, eds. Surgery of the Knee. $3^{\text {rd }}$ edn. New York, NY: Churchill Livingstone 2001: p. 913-1042.
[2] Harilainen A, Myllynen P, Antilla $\mathrm{H}$, et al. The significance of arthroscopy and examination under anaesthesia in the diagnosis of fresh injury haemarthrosis of the knee joint. Injury 1988;19(1):214.

[3] Fithian D, Paxton E, Stone ML, et al. Epidemiology and natural history of acute patellar dislocation. Am J Sports Med 2004;32(5):1114-21.

[4] Andrish JT. Recurrent patellar dislocation. In: Fulkerson JP, edr. Common patellofemoral problems. Rosemont, IL: American Academy of Orthopaedic Surgeons 2005: p. 43-55.

[5] Arendt EA, Fithian DC, Cohen E. Current concepts of lateral patella dislocation. Clin Sports Med 2002;21(3):499-519.

[6] Mäenpää H. The dislocating patella. Predisposing factors and a clinical, radiological and functional follow-up study of patients treated primarily nonoperatively. Ann Chir Gynaecol 1998;87(3):248-9.

[7] Mäenpää $H$, Huhtala $H$, Lehto MU. Recurrence after patellar dislocation. Re-dislocation in 37/75 patients followed for 6-24 years. Acta Orthop Scand 1997;68(5):424-6.

[8] Fahmy HS, Khater NH, Nasef NM, et al. Role of MRI in assessment of patello-femoral derangement in patients with anterior knee pain. The Egyptian Journal of Radiology and Nuclear Medicine 2016;47(4):148592.

[9] Conlan T, Garth WP Jr, Lemons JE. Evaluation of the medial soft-tissue restraints of the extensor mechanism of the knee. J Bone Joint Surg Am 1993;75(5):682-93.

[10] Desio DS, Burks RT, Bachus KN. Soft tissue restraints to lateral patellar translation in human knee. Am J Sports Med 1998;26(1):59-65.

[11] Hautamaa PV, Fithian DC, Kaufmann KR, et al. Medial soft tissue restraints in lateral patellar instability and repair. Clin Orthop Relat Res 1998;(349):174-82.

[12] Aichroth PM, Al-Duri Z. Dislocation and subluxation of the patella: an overview. In: Aichroth PM, Al-Duri Z, eds. Knee Surgery. London: Martin Dunitz 1992: p. 354-79.

[13] Barney L, Freeman I Recurrent dislocation. In: Campbell's Operative Orthopaedics. $7^{\text {th }}$ edn. Philadelphia: Mosby 1987: p. 2173-84.

[14] Hughston JC, Deese M. Medial subluxation of the patella as a complication of lateral retinacular release. Am J Sports Med 1988;16(4):383-8.

[15] Madigan R, Wissinger HA, Donaldson WF. Preliminary experience with a method of quadricepsplasty in recurrent subluxation of the patella. J Bone Joint Surg Am 1975;57(5):600-7.

[16] Deie M, Ochi M, Sumen Y, et al. Reconstruction of the medial patellofemoral ligament for the treatment of habitual or recurrent dislocation of the patella in children. J Bone Joint Surg Br 2003;85(6):887-90.

[17] Drez D Jr, Edwards TB, Williams CS. Results of medial patellofemoral ligament reconstruction in the treatment of patellar dislocation. Arthroscopy 2001;17(3):298-306. 
[18] Gomes JLE. Medial patellofemoral ligament reconstruction for recurrent dislocation of the patella: a preliminary report. Arthroscopy 1992;8(3):335-40.

[19] Gomes JLE, Marczyk LRS, de Cesar PC, et al. Medial patellofemoral ligament reconstruction with semitendinosus autograft for chronic patellar instability: a follow-up study. Arthroscopy 2004;20(2):147-51.

[20] Mikashima Y, Kimura M, Kobayashi Y, et al. Medial patellofemoral ligament reconstruction for recurrent patellar instability. Acta Orthop Belg 2004;70(6):54550 .
[21] Nomura E, Horiuchi Y, Kihara M. A mid-term follow-up of medial patellofemoral ligament reconstruction using an artificial ligament for recurrent patellar dislocation. Knee 2000;7(4):211-5.

[22] Nomura E, Inoue M. Surgical technique and rationale for medial patellofemoral ligament reconstruction for recurrent patellar dislocation. Arthroscopy 2003;19(5):1-9.

[23] Watanabe S, Omori G, Sofue T, et al. Short term result of medial patellofemoral ligament reconstruction for recurrent dislocation of patella. J Japan Knee Society 2004;29:167-70. 